\title{
Towards a more representative morphology: clinical and ethical considerations for including diverse populations in diagnostic genetic atlases
}

\author{
Maya Koretzky, ${ }^{1}$ Vence L. Bonham, ${ }^{2}$ Benjamin E. Berkman, ${ }^{1,3}$ Paul Kruszka, ${ }^{4}$ \\ Adebowale Adeyemo, ${ }^{5}$ Maximilian Muenke ${ }^{4}$ and Sara Chandros Hull ${ }^{1,3}$
}

\begin{abstract}
An important gap exists in textbooks (or atlases) of dysmorphology used by health-care professionals to help diagnose genetic syndromes. The lack of varied phenotypic images in available atlases limits the utility of these atlases as diagnostic tools in globally diverse populations, causing geneticists difficulty in diagnosing conditions in individuals of different ancestral backgrounds who may present with variable morphological features. Proposals to address the underinclusion of images from diverse populations in existing atlases can take advantage of the Internet and digital photography to create new resources that take into account the broad global diversity of populations affected by genetic disease. Creating atlases that are more representative of the global population will expand resources available to care for diverse patients with these
\end{abstract}

An important gap exists in the screening toolkit used by physicians and other health-care professionals to help diagnose genetic syndromes in their patients via the observation of phenotypic characteristics. Visual diagnosis relies on textbooks of dysmorphology, which include images of individuals with classic phenotypes for a wide range of genetic diseases. These morphological atlases have been a standard diagnostic tool for clinical geneticists for decades and guide clinicians in their choice of molecular testing. ${ }^{1-4}$ In the most widely used of these diagnostic atlases, the majority of the images are of individuals of northern European descent, reflecting the patient populations for whom the clinicians who developed these texts originally provided clinical care. However, because many of the genetic conditions profiled in these texts are prevalent in populations across the world, it is now clear that these texts do not sufficiently reflect global ancestral diversity. The lack of a variety of phenotypic images in available atlases potentially limits the utility of these atlases as diagnostic tools in globally diverse populations, causing geneticists difficulty in properly diagnosing conditions in individuals of different ancestral backgrounds who may present with variable morphological features. conditions, many of whom have been historically underserved by the medical system. However, such projects also raise ethical questions that are grounded in the complex intersection of imagery, medicine, history, and race and ethnicity. We consider here the benefits of producing such a resource while also considering ethical and practical concerns, and we offer recommendations for the ethical creation, structure, equitable use, and maintenance of a diverse morphological atlas for clinical diagnosis.

Genet Med advance online publication 10 March 2016

Key Words: ethics; dysmorphology; genetics; global health; diverse populations

Even the relatively simple diagnosis of Down syndrome in diverse populations is not straightforward, as seen in Figure 1, which includes photographs of children with Down syndrome from understudied populations. The observer's gestalt conclusion upon examining these photos may not be the diagnosis of Down syndrome, because the eye and nose differences that occur in various ethnicities mask the "textbook" features described in most medical texts. For example, a feature of Down syndrome is epicanthal folds at the inner portion of the eye; however, this is a normal finding in individuals of Asian descent. This difficulty with diagnosis is not unique to countries with diverse racial and ethnic populations; diagnosis can be challenging even in locations with relatively homogeneous populations if this majority is of non-European ancestral origin. Skilled local clinicians working in these areas may still struggle to identify genetic syndromes by phenotype, because the available training tools and classical phenotype images of particular disorders predominantly feature individuals with European ancestry.

Proposals to address the underinclusion of images of human malformation syndromes from diverse populations in existing morphological atlases, such as the one described by Muenke and

\footnotetext{
${ }^{1}$ Department of Bioethics, Clinical Center, National Institutes of Health, Bethesda, Maryland, USA; ${ }^{2}$ Social and Behavioral Research Branch, National Human Genome Research Institute, National Institutes of Health, Bethesda, Maryland, USA; ${ }^{3}$ Bioethics Core, National Human Genome Research Institute, National Institutes of Health, Bethesda, Maryland, USA; ${ }^{4}$ Medical Genetics Branch, National Human Genome Research Institute, National Institutes of Health, Bethesda, Maryland, USA; ${ }^{5}$ Center for Research on Genomics and Global Health, National Human Genome Research Institute, National Institutes of Health, Bethesda, Maryland, USA.Correspondence: Sara Chandros Hull (shull@mail.nih.gov)
} 

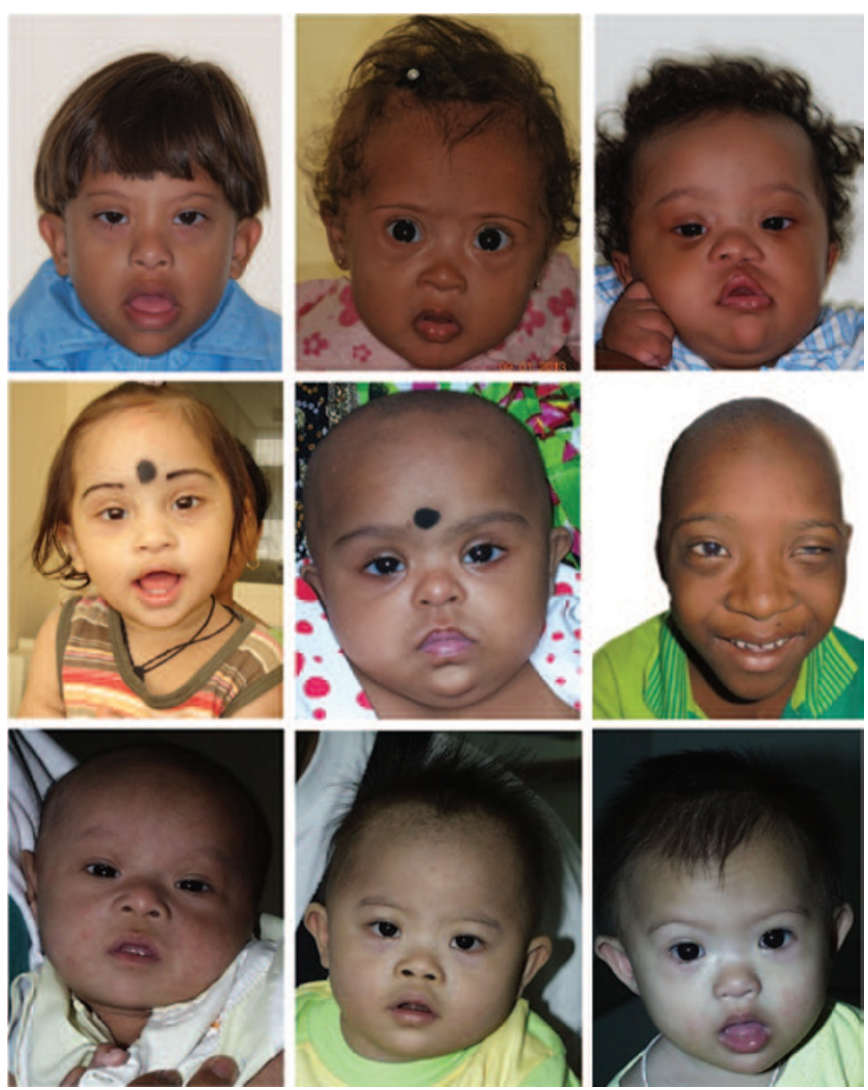

Figure 1 Children with Down syndrome from Thailand, India, and Nigeria. Courtesy of Ekanem Ekure, S.J. Patil, Girisha K.M., Antonio RichieriCosta, and Vorasuk Shotelersuk.

colleagues in this issue of Genetics in Medicine, ${ }^{5}$ can take advantage of the ubiquity of the Internet and the ease of digital photography to create new resources that take into account the broad global diversity of populations affected by genetic disease and can be made widely available. Creating a genetic dysmorphology atlas that is more representative of the global population will help expand the resources available to care for diverse patients with these conditions, many of whom have been historically underserved in various ways by the medical system.

Birth defects are now a leading cause of childhood mortality and morbidity worldwide; however, medical geneticists are most commonly found in university medical centers in developed countries. Most developing countries do not have medical geneticists. For example, although the largest country in Africa is Nigeria, with more than 200 million people and the largest economy, several of the authors of this article (A.A., P.K., M.M.) travel there regularly and are aware that there are no practicing clinical geneticists there; our collaborators in Nigeria are pediatric cardiologists. This is contrast to the Washington, DC, area, where there are more than 20 medical (MD) geneticists.

From a clinical perspective, more diverse atlases would enable more accurate and earlier syndromic diagnosis of congenital malformations to be made across patients of a variety of ancestral origins, potentially leading to improved medical care for persons of non-European descent with these conditions. ${ }^{6}$
Including a wider selection of individuals in morphological atlases could also lay the groundwork for addressing other aspects of medical diagnosis and care, as well as genetic disease, by building relationships and research capacity in the international arena. ${ }^{7}$ For example, allowing international physicians to participate in the creation of the atlas by contributing images of their patients can facilitate international cooperation and establish networks of clinicians and researchers in underresourced areas. By enabling more accurate diagnosis of individuals, the atlas would also enable researchers to aggregate these data and glean a more accurate picture of the global prevalence of currently underdiagnosed genetic diseases ${ }^{8}$ (for instance, evidence is emerging that cystic fibrosis is vastly underdiagnosed in populations of non-European origin ${ }^{9}$ ).

However, the project also raises ethical questions about the selection and portrayal of individuals in the atlas and who will have access to this database. We consider the benefits of producing such a resource and consider the ethical and practical concerns raised. We also offer recommendations for the ethical creation, structure, equitable use, and maintenance of a diverse morphological atlas for clinical diagnosis.

\section{ETHICAL CONSIDERATIONS}

The ethical and social concerns that are raised by the creation of a diverse morphological atlas are grounded in the complex intersection of imagery, medicine, history, and race and ethnicity. These concerns can be sorted into two general categories: (i) historically rooted concerns about reifying racial and ethnic groups as discrete biological classifications and the misuse of racial and ethnic categories and (ii) contemporary considerations regarding access to the database and respecting patient autonomy and privacy in an Internet-based environment.

There is a long and complex history of classifying people into groups to search for a biological basis for racial difference, ${ }^{10,11}$ with race persistently occupying a liminal space between social construct and biological utility. ${ }^{12}$ The medicalization of race has at times been used as a way to justify discriminatory practices outside of the medical sphere and as a way to challenge these same practices and push back against them. ${ }^{13}$ This complex relationship between medicine and race politics has been demonstrated by scholars in various contexts, from Lundy Braun's classic work on differing spirometry measures for different races to Nancy Pollock's scholarship on the history and anthropology of how cardiac disease in African Americans has been articulated by the medical establishment. ${ }^{14}$ (For one example regarding heart disease, see ref: 15.) In many ways, biomedicine continues to reify an interpretation of race as a biological quality that affects health and disease, rather than as an identity that is more indicative of social group and environmental influences than of an underlying physiology.

Although the notion of "biological race" has been misused by medical professionals in many instances, it is also true that disease and health risks facing different individuals may be tracked by ancestry, social experiences, and environmentqualities for which race identification may often act as a proxy. ${ }^{16}$ 
Scholars such as David Wasserman and Nancy Krieger, among others, have explored the benefits and pitfalls of using race categories in medicine for individual therapeutic purposes or as a way to talk about social groups and structural racism and their impact on health. ${ }^{17,18}$

This tension between the medical utility and the medical misuse of race categories is perhaps most salient in the field of genetics, a discipline in which disease risks are correlated with ancestry but that also has historical ties to nineteenth- and twentieth-century eugenics science. ${ }^{19}$ Scholars from a wide range of disciplines have explored the ethics, anthropology, and history of race and genetics. (For further reading, please see refs. 20-22.) The language used by geneticists may serve to confuse the connections that exist between race and genetics. Although genetic variation correlates with ancestral markers, race is a much more complex and largely self-identified concept that cannot be determined through biomedical testing. ${ }^{23,24}$ Although guidelines on the use of accurate terminology related to race categories in genetic research publications emphasize the importance of defining how these terms are being used in a given research project (e.g., as a proxy for ancestral origin or socioeconomic status), these guidelines are rarely followed in contemporary genetics publications. ${ }^{25}$

Medical geneticists have often been in the middle of racedysmorphology debates. Indeed, they were instrumental in changing one of its more explicit expressions: referring to children with trisomy 21 as "mongols" or as having "mongoloid features" in medical literature as well as in the lay press. This practice originated in the 1860 s, when a physician noted physical similarities in the appearance of people with trisomy 21 and individuals previously described as belonging to the "Mongoloid" race. Nearly a century later, geneticists began to call for discontinuing the use of the term "mongoloid" and its variants, opting for the term "Down syndrome" instead. Although the term remains in use, geneticists and professional organizations have formally recognized that terminology that connects a genetic condition associated with cognitive impairment, physical features of that condition, and a country, such as Mongolia, is derogatory and should be discontinued. ${ }^{26}$

In addition to these important linguistic nuances, there is also a long history of the unjust use of morphological images of different racial "types" by the biomedical establishment. The populations that have been left out of morphological diagnostic atlases are also populations that have historically been underserved or exploited by the medical professions. In many cases, racialized images played a large role in pseudoscientific research to bolster claims of biological differences between races and to argue for the premise of inferiority of certain groups. ${ }^{27}$ For example, throughout the nineteenth century, the widespread creation of eugenicist atlases and, later, Nazi photographic databases were used for cataloguing and distinguishing between the races, with the ultimate goal of eradicating populations believed to be inferior. ${ }^{28}$ Images of disabled individuals also figured prominently in medically sanctioned eugenics campaigns well into the twentieth century in both America and Western Europe ${ }^{29}$.
Although the goals of this project are wholly different from these historical analogues, the very practice of organizing racially labeled images in a medical textbook merits careful scrutiny because of this long and problematic history.

We also considered concerns that have been expressed by contemporary disability rights advocates that by reducing an individual's facial features or distinctive physiological traits to a set of symptoms for determining a genetic diagnosis, dysmorphology atlases might objectify the individual being photographed and could reinforce stereotypes and stigma associated with these conditions. (See, for example, ref. 29.) Although these are important issues to consider, we decided that adequate treatment of disability perspectives is beyond the scope of this article.

In addition there are several important practical decisions to work through regarding the database. For example, who will maintain the database and how will access be regulated? An open-access database would be consistent with the justiceoriented goals of the project to expand the availability of such diagnostic tools around the world. However, the easy accessibility of race-labeled images and data could pose risks such as stigmatizing a particular individual, community, or population..$^{30}$ The database could potentially be accessed to use the images or other material wrongfully and with malicious intent. This tension between the ideal of open-access medical knowledge and the practical realities of patient privacy is not speculative; similar concerns have begun to emerge for other online platforms for uploading, distributing, and viewing ostensibly anonymized patient images by medical professionals. ${ }^{31}$ However, we feel that the goals of this project to assist medical providers in making earlier and more accurate diagnoses of dysmorphic syndromes (both environmentally and genetically caused) will provide a benefit that outweighs such concerns about widespread access.

In addition to patient privacy, there are other important questions raised by this database at the level of individual participants. For example, it may be challenging to obtain the informed consent of individual participants to generate and use their images in the atlas for several reasons. Participant families who come from especially remote or underresourced areas may have difficulty understanding the scope of who will be able to access the images. Craniofacial defects require the inclusion of facial pictures for a diagnosis, which increases the risk of individuals being identified. Because many of the diseases that will be profiled manifest in childhood and/or are characterized by cognitive impairment or intellectual disability, many participants will be unable to formally consent on their own behalf, which raises questions about surrogate permission and whether it is sufficient for use of their images. Furthermore, there are questions about whether it will (and should) be possible for participants to remove their images from the database if they change their mind about participation in the project. These questions raise important concerns about autonomy and privacy for individuals who will be photographed and included in the database. 


\section{RECOMMENDATIONS}

Taking these historical concerns and ethical questions seriously will require careful design and implementation decisions in the creation of this kind of resource (Table 1). Especially important when considering the historical context of morphological atlases are questions of how ancestry will be determined for the purposes of this project, how it will be noted in the atlas, and how individuals of different ancestral backgrounds will be selected to participate. The goal is to strike a balance between providing useful data to local clinicians (for example, clinicians working in only one country may want to be able to narrow the atlas to only show patient images from that country) and avoiding the reification of racial or ethnic categories. Our recommendations are informed by engagement with an international group of advisors who are clinicians from non-Western countries, including Uganda, Nigeria, South Africa, Rwanda, Mali, Malaysia, India, Thailand, Japan, China, South America, and the Middle East. Both the necessity of new atlases and the structure of the project are grounded in challenges and complexities that were identified by members of this diverse group who are providing oversight of the website described by Muenke et al. ${ }^{5}$

We believe that the organization of newer atlases can be an important point of departure from the problematic historical examples discussed. Atlases should be structured to allow sorting of images by disease or by the current country or region of residence of the photographed patient so that clinicians could search for all individuals with a particular condition (e.g., any patient with Williams syndrome) and/or by nation or region (e.g., all photographs of individuals living in Sub-Saharan Africa). Patients would then be given the opportunity to identify subjective ethnic, racial, and/or tribal identities that would appear alongside the picture of the individual in addition to data about the national origins of each of the subject's four grandparents, but they would not be searchable variables in the database. For example, the photograph of a Southeast Asian individual with Down syndrome will appear on the screen if "Down syndrome" or "Southeast Asia" is searched. If the subject self-identified as Khmer and indicated that all four grandparents were born in Thailand, then this information will appear with the image of the subject as additional descriptive information, but it will not be possible for clinicians to search the database by the term "Asian," "Khmer," or "Thai" ancestral origin. Our work draws on the model of language for genetic variation and ancestry proposed previously. ${ }^{32}$

By organizing the searchable features of the atlas by disease or current nationality, and by associating self-identified ethnic/ racial/tribal identity information with the images in a nonsearchable manner, the clinical utility of the atlas as a diagnostic tool across a wide range of phenotypes can be realized while limiting the possible unintended uses of such data for questions related to eugenic, scientifically misplaced, or other flawed research. The standard method of tracing ancestral origin via self-report of the nationalities of a participant's four grandparents seems to be an appropriate method for morphological atlases as well. We acknowledge that estimating ancestral origin via one's four grandparents may be less informative in some populations with significant diversity of continental ancestry, for example, within the United States. ${ }^{33}$ However, we emphasize that the goals of the new atlas are to be inclusive of populations from around the world, most of whom will tend not to have the same degree of heterogeneity of continental ancestry.

Another important way that new atlases can be distinguished from historical projects involving the classification of individuals by race is to include individuals from a geographically diverse population while avoiding the use of ethnicity-related criteria and language when selecting participants and categorizing them for the atlas ${ }^{25}$ by selecting participants from the pool of individuals who arrive at participating medical centers around the world. The goal of the atlas is to reflect the diversity that is seen in clinical practice around the world rather than to parse the origin of patients by self-reported "race," continental ancestry, or ethnic origin. Following diagnosis of a genetic disease, they will be approached about participating in the atlas project first, and asked for their ancestral origin data later in the process. No quota of individuals of different ancestral backgrounds to be included in the atlas will be set, nor will attempts be made to find "pure" examples of different ancestral groups. Treating ancestry as data to be collected after participants are selected, asked for their consent, and photographed will help to guard against the selection of subjects who are typical ("pure"

\section{Table 1 Summary of recommendations for the creation of new diverse morphological databases}

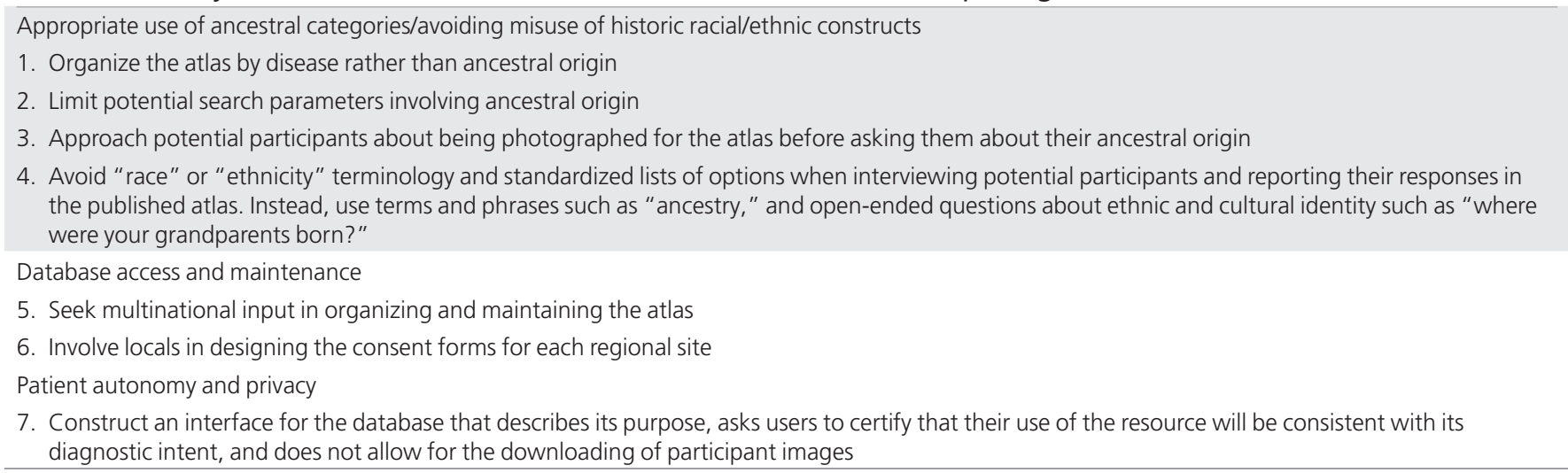


or "ideal") types of any given group, thus distancing this project from prior eugenics-oriented image collecting practices.

Additionally, we recommend that participants should not be prompted to provide data regarding their ancestral origins from a pre-existing list of possible race or ethnic categories, such as the list suggested by the US Office of Management and Budget (https://www.whitehouse.gov/omb/fedreg_race-ethnicity). Additionally, these categories do not reflect how individuals outside the United States describe themselves. The terms contained in such lists are generally insufficient for capturing the genetic diversity of human populations and do not accurately describe the goal of an atlas that aims to represent individuals with a wide variety of ancestral backgrounds. In making this recommendation, we are following the guidelines set by the Race, Ethnicity, and Genetics Working Group of the American Journal of Human Genetics. ${ }^{12}$ At best, these terms included in such lists are proxies for constructs that can be more accurately captured through other data, such as the continental origins of four grandparents. In addition, as described, they carry multiple connotations (social, biological, cultural), many of which are inaccurate or simply not relevant to this endeavor.

The question of what person, group, or organization will collect and maintain the images is an important one that is tied to concerns about the potential exploitation of marginalized groups. Although the United States has resources and expertise to contribute to this initiative, a purely US-led approach would undermine the goal of having this be a more diverse and accessible resource with buy-in from the various communities involved. An advisory board that includes representatives from the continental regions from which clinicians will solicit images to oversee the project and the database will provide a more appropriate mechanism for defining the agenda, goals, and implementation of this international project. ${ }^{34}$ Involving local physicians and community members in the design of the database will help to craft the resource in a way that maximizes the benefits and minimizes the harms to the diverse communities in which it will be used. In an effort to prevent local power relationships from affecting the collection of the data, all collaborators will be briefed on how to properly ascertain ancestry information, focusing on medical conditions and ancestral geographic origins.

A widely accessible morphological database that is freely available on the Internet is important for both justice and transparency reasons. However, it also raises the possibility of undesirable uses and unintended consequences of an open-access morphological database that are causes for concern. Requiring users to access the database via a screen that describes the intended uses of the database and to electronically sign a form agreeing to terms of use should help to mitigate this concern, although it does not provide a mechanism for enforcement of these terms. Unintended uses can also be minimized by digitally protecting images so that they cannot be downloaded from the website and, as mentioned, so that sorting images by ancestral origin alone be prevented.
The recommended multinational oversight structure should be consulted to design an appropriate consent process for individuals who participate in the project. Practically speaking, the consent process needs to address the specific informational needs of each community involved (for example, local literacy levels and educational attainment). ${ }^{35} \mathrm{~A}$ well-designed, transparent consent process that is vetted by local leadership and local regulatory experts is more likely to be perceived as trustworthy by individual participants and their communities. In addition to their involvement in writing consent forms, local collaborators should also be directly involved in identifying appropriate participants within their locales. ${ }^{36}$

There are additional considerations regarding the informed consent process that require further exploration. The process will need to inform potential participants about the privacy risks associated with publishing images and other data on the broad and international scale that is proposed for this project, and it will need to sufficiently address any relevant privacy regulations that govern the sharing of medical images and information via the Internet. Given the young age of many of the potential subjects in the atlas, an appropriate mechanism for parental authorization will be an important component of this project as well. Similarly, a surrogate mechanism to authorize the participation of adults with limited cognitive capacity, as is characteristic of many of the conditions that will be included in the atlas, will be necessary. Decisions also need to be made about whether participants will be offered the choice to withdraw their images from the database at a later date if they so choose, or whether their ability to withdraw will be limited once the images have been published in this resource.

\section{CONCLUSION}

The creation of new morphological atlases that take into account the broad diversity of the populations affected by genetic diseases is an important step in extending the benefits of medical genetics to the global populations who are currently underserved. Knowing that a child has a particular syndromic diagnosis can be lifesaving by providing important information about other significant organ systems that are often affected. In addition, an early and accurate diagnosis can enable physicians to perform appropriate preventative care and give affected families an idea of what lies ahead. At the same time, there are a number of ethical considerations that should be addressed in any project that relies on the publication of images and genetic information from persons with dysmorphic features who come from a variety of ancestral backgrounds. Our goals in this paper were both constructive and preventive in nature, providing recommendations to guide the creation of a maximally beneficial resource while also mitigating potential problems with the project before they arise. Ultimately, we believe that the ethical concerns that have been identified, although serious, are outweighed by the potential benefits to populations who have not been included in such resources to date, and that appropriate steps can be taken to mitigate these ethical concerns. 


\section{ACKNOWLEDGMENTS}

This work was funded in part by the intramural research program of the National Human Genome Research Institute. The authors acknowledge the helpful feedback of Donald Hadley, Manjit Kaur, and participants at the Mid-Atlantic Bioethics Fellows' Colloquium for earlier versions of this work. Written parental consent was obtained to publish the photographs of children with Down syndrome that are included in Figure 1.

The views expressed are the authors' own. They do not represent the position or policy of the National Institutes of Health, the US Public Health Service, or the Department of Health and Human Services.

\section{DISCLOSURE}

The authors declare no conflict of interest.

\section{REFERENCES}

1. Clarren, SK. Book review. Atlas of Clinical Syndromes: A Visual Aid to Diagnosis. N Engl J Med 1992;327:739-740.

2. Reardon W. The Bedside Dysmorphologist. Oxford University Press: New York, 2007.

3. Wiedemann HR, Kunze J, Dibbern H. Atlas of Clinical Syndromes: A Visual Aid to Diagnosis. 2nd edn. Mosby-Year Book: St. Louis, MO, 1992.

4. Baraitser M, Winter R (eds.). Colour Atlas of Congenital Malformation Syndromes. Mosby-Wolfe: London, 1996.

5. Muenke M, Adeyemo A, Kruszka P. An electronic atlas of human malformation syndromes in diverse populations. Genet Med E-pub ahead of print 3 March, 2016.

6. Tekendo-Ngongang C, Sophie D, Seraphin N, Stefania G, Sloan-Béna F, Wonkam A. Challenges in clinical diagnosis of Williams-Beuren syndrome in sub-Saharan Africans: case reports from Cameroon. Mol Syndromol 2014;5:287-292.

7. Zusevics KL. Public health genomics: a new space for a dialogue on racism through Community Based Participatory Research. Public Health 2013;127:981-983.

8. Lim RM, Silver AJ, Silver MJ, et al. Targeted mutation screening panels expose systematic population bias in detection of cystic fibrosis risk. Genet Med 2016;18:174-179.

9. Bobadilla, JL, Macek M, Fine JP, Farrell PM. Cystic fibrosis: a worldwide analysis of CFTR mutations--correlation with incidence data and application to screening. Hum Mutat 2002;19:575-606.

10. Yudell M. Race Unmasked Biology and Race in the 20th Century. Columbia University Press: New York, 2014.

11. Marks J. Human Biodiversity: Genes, Race and History. Aldine de Gruyter: New York, 1995.

12. Race, Ethnicity, and Genetics Working Group. The use of racial, ethnic, and ancestral categories in human genetics research. Am J Hum Genet 2005;77:519-532.

13. Braun L, Fausto-Sterling A, Fullwiley D, et al. Racial categories in medical practice: how useful are they? PLoS Med 2007;4:e271.

14. Braun L. Breathing Race into the Machine. University of Minnesota Press: Minneapolis, MN, 2014.

15. Pollock, A. Medicating Race: Heart Disease and Durable Preoccupations with Difference. Duke University Press: Durham, NC, 2012.
16. Bamshad M. Genetic influences on health: does race matter? JAMA 2005;294:937-946.

17. Wasserman, D. The justifiability of racial classification and generalizations in contemporary clinical and research practice. Law Prob Risk 2010;3-4: 215-226.

18. Krieger N. Stormy weather: race, gene expression, and the science of health disparities. Am J Public Health 2005;95:2155-2160.

19. Comfort, N. The Science of Human Perfection: How Genes Became the Heart of American Medicine. Yale University Press: New Haven, CT, 2014.

20. Bliss, C. Race Decoded: The Genomic Fight for Social Justice. Stanford University Press: Stanford, CA, 2012.

21. Lee SS, Koenig BA, Richardson SS (eds.). Revisiting Race in a Genomic Age. Rutgers University Press: New Brunswick, NJ, 2008.

22. Reardon, J. Race to the Finish: Identity and Governance in an Age of Genomics: Identity and Governance in an Age of Genomics. Princeton University Press: Princeton, NJ, 2009.

23. Kahn, J. Genes, race, and population: avoiding a collision of categories. Am J Public Health 2006;96:1965-1970.

24. Duster, T. Race and reification in science Science 2005;307:1050-1051.

25. Sankar P, Cho MK, Monahan K, Nowak K. Reporting race and ethnicity in genetics research: do journal recommendations or resources matter? Sci Eng Ethics 2015;21:1353-1366.

26. Orr, G. Why are the words 'mongol,' 'mongoloid,' and 'mongy' still bandied about as insults? Independent 23 November 2014 . http://www.independent. co.uk/arts-entertainment/tv/features/why-are-the-words-mongol-mongoloidand-mongy-still-bandied-about-as-insults-9878557.html. Accessed 30 June 2015.

27. Blumenbach JF, The Anthropological Treatises of Johann Friedrich Blumenbach. Longman, Roberts \& Green: London, 1865.

28. United States Holocaust Memorial Museum. Deadly Medicine: Creating The Master Race. United States Holocaust Memorial Museum: Washington, DC, 2004.

29. Wilson JC. Making disability visible: how disability studies might transform the medical and science writing classroom. Tech Comm Quart 2000;9: 149-161.

30. Parr, H. New body-geographies: the embodied spaces of health and medical information on the internet. Environ Plann D 2002;20:73-95.

31. Virginia H. Is this doctors app a digital classroom—or medical porn? BuzzFeed News. http://www.buzzfeed.com/virginiahughes/is-this-doctors-app-a-digitalclassroom-or medical-form/. Accessed 22 May 2015.

32. Sankar P, Cho MK. Genetics. Toward a new vocabulary of human genetic variation. Science 2002;298:1337-1338.

33. Yaeger R, Avila-Bront A, Abdul K, et al. Comparing genetic ancestry and selfdescribed race in african americans born in the United States and in Africa. Cancer Epidemiol Biomarkers Prev 2008;17:1329-1338.

34. Lantz PM, Viruell-Fuentes E, Israel BA, Softley D, Guzman R. Can communities and academia work together on public health research? Evaluation results from a community-based participatory research partnership in Detroit. J Urban Health 2001;78:495-507.

35. Buseh AG, Stevens PE, Millon-Underwood S, Townsend L, Kelber ST. Community leaders' perspectives on engaging African Americans in biobanks and other human genetics initiatives. J Community Genet 2013;4: 483-494.

36. Polanco FR, Dominguez DC, Grady C, et al. Conducting HIV research in racial and ethnic minority communities: building a successful interdisciplinary research team. J Assoc Nurses AIDS Care 2011;22:388-396. 